\title{
A tacholess order tracking methodology based on a probabilistic approach to incorporate angular acceleration information into the maxima tracking process
}

\author{
S. Schmidtt ${ }^{\mathrm{a}, *}$, P.S. Heyns ${ }^{\mathrm{a}}$, J.P. de Villiers ${ }^{\mathrm{b}, \mathrm{c}}$ \\ ${ }^{a}$ Centre for Asset Integrity Management, Department of Mechanical and Aeronautical Engineering, University of \\ Pretoria, Pretoria, South Africa \\ ${ }^{b}$ Department of Electrical, Electronic and Computer Engineering, University of Pretoria, Pretoria, South Africa \\ ${ }^{c}$ Defence, Peace, Safety and Security (DPSS), Council for Scientific and Industrial Research (CSIR), PO Box \\ 395, Pretoria 0001, South Africa
}

\begin{abstract}
Rotational speed or phase measurements are important in the rotating machine condition monitoring field. Rotating machines often operate in the presence of fluctuating rotational speeds and its components generate signals which are periodical in the angle domain. Hence, it is essential to perform order tracking when condition monitoring is performed under varying speed conditions. Computed order tracking is performed with a measured tachometer signal and it requires additional hardware to be installed on the machine, which may not be feasible nor practical. This article presents a tacholess order tracking method which is capable of accurately estimating the phase of a shaft of interest in the presence of large angular accelerations and noise. An improved maxima tracking procedure is used with an angular-displacement Vold-Kalman filter and the Hilbert transform to estimate the instantaneous phase of the shaft under consideration. The estimated instantaneous phase is used to resample the vibration signal from the time to the angle domain. The proposed tacholess order tracking technique is critically investigated on three numerical and three experimental rotational speed profiles. The minimum and maximum phase error obtained for the experimental data was 0.037697 and 0.05022 radians respectively, which highlights the potential of the technique.
\end{abstract}

\section{Keywords:}

Tacholess order tracking, Maxima tracking, Vold-Kalman filter, Rotational speed estimation, Probabilistic, Linear regression

\footnotetext{
${ }^{*}$ Corresponding author.

Email address: stephanschmidt@zoho.com (S. Schmidt)
} 


\section{Introduction}

Rotating machines, such as gearboxes, operate under fluctuating operating conditions due to inherent fluctuating characteristics, such as fluctuating wind speeds for wind turbines, that are present during its operation. The resulting vibration signal, measured on a rotating machine, is best represented in the angle domain, as opposed to the time domain, since the information within the signal such as localised gear faults and gear mesh interactions are generated at specific angular positions on the shaft [1].

The vibration signal is transformed from the time to the angle domain using order tracking techniques, where the instantaneous phase of the shaft of interest is used to resample the vibration signal into equal angular increments. In computed order tracking, a tachometer signal is measured simultaneously with the vibration signal by using a stationary probe and a reference, such as an incremental shaft encoder or a shaft key, on the shaft of interest. This tachometer signal contains the information pertaining to the instantaneous phase of the shaft, which is used to perform order tracking on the vibration signal. The order tracking process has been successfully applied for gear fault detection [2, 3], misalignment fault diagnosis [4] and bearing fault diagnosis [5].

Single pulse per revolution (PPR) tachometer signals, obtained from a shaft key and a proximity probe, provide insufficient resolution to capture large angular accelerations. Incremental shaft encoders, such as zebra tape and digital shaft encoders, provide much finer resolutions to measure the large angular accelerations in the system [6]. However, incremental shaft encoders require large sampling frequencies to accurately estimate the zero crossing times $[7,8]$, the procurement and installation of additional expensive instrumentation and equipment and the storage of additional data $[9,10]$. The shaft encoders are also in some cases impractical or even impossible to install and are not durable in the harsh operating conditions [11, 12]. The vibration transducer and the optical probe are located on different components and therefore the transmission paths between the measurement equipment and the source of the vibration are different [13]. This transmission path is frequency dependent and leads to phase distortion when performing computed order tracking in varying speed conditions [13, 14]. Tacholess order tracking approaches, which use the acceleration signal to obtain the instantaneous phase information, are therefore actively investigated by the engineering community to circumvent the aforementioned 
limitations and problems when performing computed order tracking [4, 9, 10, 12, 15].

Bonnardot et al. [8] proposed using the acceleration signal to perform tacholess order tracking in slightly varying rotational speed applications using narrow-band demodulation on one of the gear mesh frequencies. Combet and Gelman [11] improved the procedure proposed by Bonnardot et al. [8], by automating the harmonic selection process. Heyns et al. [16] used a clustering procedure to track an instantaneous frequency component in the spectrogram of the vibration signal. The IF estimate is low-pass filtered, since it contains noise due to the limited resolution provided by the spectrogram. The filtered instantaneous frequency is used to resample the signal for fault detection.

Urbanek et al. [10] and Zhao et al. [12] proposed tacholess order tracking procedures in large varying speed conditions. Urbanek et al. [10] proposed performing maxima tracking on the spectrogram of the vibration signal. The rough estimate of the instantaneous frequency, obtained from the maxima tracking process, is used to order track the signal and filter out all components except a single instantaneous frequency. This mono-component signal is reverse resampled to the time domain. Zhao et al. [12] used the Chirplet transform to extract an instantaneous frequency component and then the angular-velocity Vold-Kalman filter is applied to the signal to obtain a mono-component signal. The Hilbert transform is used on the mono-component signal by Urbanek et al. [10] and Zhao et al. [12] to extract the instantaneous phase of the signal over time. This instantaneous phase provides a mapping from the time to the angle domain, which is used to order track the vibration signal.

Qi et al. [4] used the ensemble empirical mode decomposition algorithm to obtain the monocomponent intrinsic mode functions of the signal, which are subsequently used to obtain the phase information of the shaft of interest. He et al. [17] proposed using the discrete spectrum correction technique to order track the vibration signal obtained from a wind turbine. Leclere et al. [9] proposed a multi-order approach for instantaneous angular speed estimation. The spectrogram is applied to the vibration signal, afterwhich a whitening procedure is applied to attenuate structural resonances. The probability density function (pdf) within an expected operating frequency range is estimated at each time step using multi order information, whereafter the pdf is smoothed to calculate the instantaneous angular speed.

In this study, a tacholess order tracking method is proposed which is robust to large varying 
speed conditions and in the presence of noise. The estimated rotational speed is investigated as well, because this information is invaluable for condition-based maintenance decision-making purposes. The benefits of incorporating acceleration information into the process is highlighted, which can possibly be used to develop multi-order approaches. The tacholess order tracking technique is developed in section 2 and validated in section 3, whereafter conclusions are drawn and recommendations are made in section 4 .

\section{Proposed tacholess order tracking methodology}

The proposed tacholess order tracking method, illustrated in Figure 1, is very similar to the methods proposed by Urbanek et al. [10] and Zhao et al. [12]. In the proposed tacholess order tracking method, the maxima tracking algorithm is improved by incorporating acceleration information into the maxima tracking process which renders it more robust in the presence of angular acceleration and noise components. The angular-displacement Vold-Kalman filter is investigated as opposed to the angular-velocity Vold-Kalman filter used by [12].

The rotational speed profile, $f_{r}$ versus time, and the acceleration signal, abbreviated by acc., are presented in (a) of Figure 1. The spectrogram of the vibration signal in (a), is presented in (b). An instantaneous frequency (IF) component is tracked with the proposed maxima tracking procedure to identify the relationship between the IF and time. The tracked IF component is superimposed on the spectrogram in (c).

The tracked instantaneous frequency in (c) of Figure 1 is used as the centre frequency for an angular-displacement Vold-Kalman filter (VKF), discussed in section 2.3. The VKF is a bandpass filter with a time-varying centre frequency. The spectrogram of the filtered vibration signal is shown in (d), where it can be seen that it is a mono-component signal only containing the tracked IF. The phase of this mono-component signal is calculated from the Hilbert transform and shown in (e). Note that the phase of the IF needs to be scaled to reflect the phase of the shaft of interest. The phase of the shaft of interest, presented in (f), is used to order track the vibration signal. The ordinate (or phase of the shaft) in Figure (f) is sampled with equal angle lengths and then new time sampling points are determined from the instantaneous phase profile. Each step of this process is discussed in more detail in the subsequent subsections. 


\section{Nomenclature}

\begin{tabular}{|c|c|c|c|}
\hline$\beta$ & Envelope of signal of interest & $e$ & Phase error [rad] \\
\hline $\boldsymbol{A}$ & Weight matrix & $e_{R}$ & Relative phase error \\
\hline$Q$ & Design matrix & $f_{b}$ & Bandwidth $[\mathrm{Hz}]$ \\
\hline$\Delta f_{\Gamma}$ & $\begin{array}{l}\text { Frequency resolution of spectrogram } \\
{[\mathrm{Hz}]}\end{array}$ & $f_{c}$ & Centre of the constraint $[\mathrm{Hz}]$ \\
\hline$\Delta f_{c}$ & $\begin{array}{l}\text { Feasible bandwidth enforced by the } \\
\text { constraint }[\mathrm{Hz}]\end{array}$ & $\begin{array}{l}f_{F} \\
f_{s}\end{array}$ & $\begin{array}{l}\text { A polynomial model of the IF }[\mathrm{Hz}] \\
\text { Sampling frequency }[\mathrm{Hz}]\end{array}$ \\
\hline $\begin{array}{l}\Delta t \\
\Delta t_{\Gamma}\end{array}$ & $\begin{array}{l}\text { Sampling period }[\mathrm{s}] \\
\text { Time resolution of spectrogram }[\mathrm{s}]\end{array}$ & $f_{\max }$ & $\begin{array}{l}\text { The IF estimated from maxima track- } \\
\text { ing }[\mathrm{Hz}]\end{array}$ \\
\hline$\Gamma$ & Short-time Fourier transform & $f_{\mathrm{IF}}$ & The actual, unknown IF $[\mathrm{Hz}]$ \\
\hline$\kappa$ & Cost function & $f_{r}$ & Rotational speed of shaft $[\mathrm{Hz}]$ \\
\hline$\lambda_{i}$ & Lagrangian multiplier of constraint $i$ & $H$ & The Hilbert transform \\
\hline $\mathcal{L}$ & Lagrangian function & $j$ & Imaginary unit \\
\hline$\nu$ & A sample from a Gaussian noise model & $L_{o}$ & Window overlap [number of samples] \\
\hline$\phi_{a}$ & Analytical phase of the shaft [rad] & & \\
\hline$\phi_{r}$ & Estimated phase of the shaft [rad] & $L_{w}$ & Vindow length [number of samples] \\
\hline$\phi_{\mathrm{IF}}$ & Phase of instantaneous frequency [rad] & $L_{z}$ & Zero padding [number of samples] \\
\hline $\begin{array}{l}\rho_{i} \\
\sigma\end{array}$ & $\begin{array}{l}\text { Weight parameter of constraint } i \\
\text { Standard deviation }\end{array}$ & $N_{m}$ & $\begin{array}{l}\text { Number of data points used for linear } \\
\text { regression }\end{array}$ \\
\hline$v$ & Window function & $N_{p}$ & Order of the polynomial \\
\hline$\varepsilon$ & Non-homogeneity term & $N_{x}$ & Number of samples in $x$ \\
\hline$\zeta$ & $\begin{array}{l}\text { Residual component of the data equa- } \\
\text { tion }\end{array}$ & $p$ & Probability density function \\
\hline$a$ & Weighting factor & $s_{i}$ & ariable of constraint $i$ \\
\hline$B_{w}$ & Relative bandwidth & $t$ & \\
\hline$c_{s}$ & $\begin{array}{l}\text { Fundamental component w.r.t shaft } \\
\text { orders }\end{array}$ & $\begin{array}{l}w_{i} \\
x\end{array}$ & $\begin{array}{l}\text { Polynomial weight coefficient } i \\
\text { The vibration signal }\end{array}$ \\
\hline
\end{tabular}

\subsection{Time-frequency distribution}

The time and frequency domain information in the vibration signal are investigated simultaneously in a time-frequency distribution so that the IF of interest can be estimated at each time step. Urbanek et al. [10] used the spectrogram for maxima tracking and Zhao et al. [12] used the spectrogram to estimate the chirp rate so that the Chirplet transform could be used to esti- 
(a)
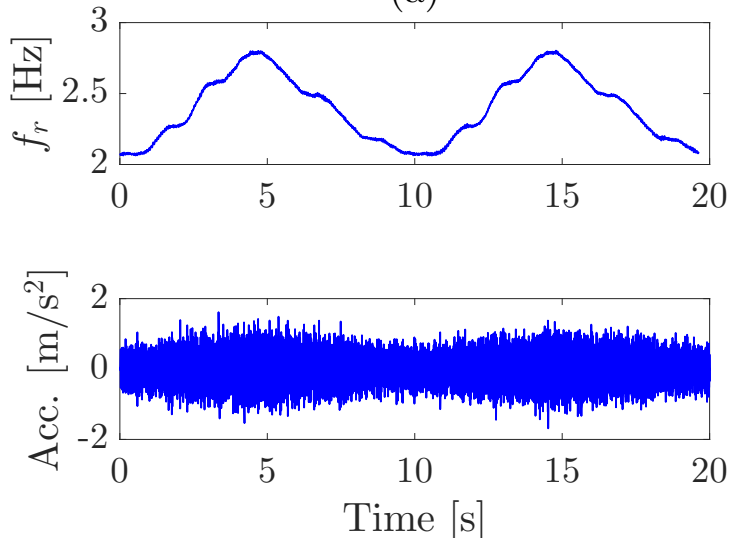

(c)

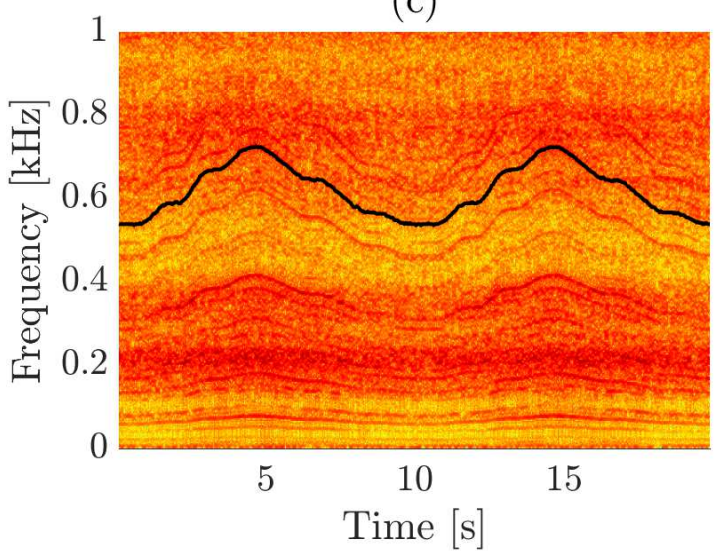

(e)

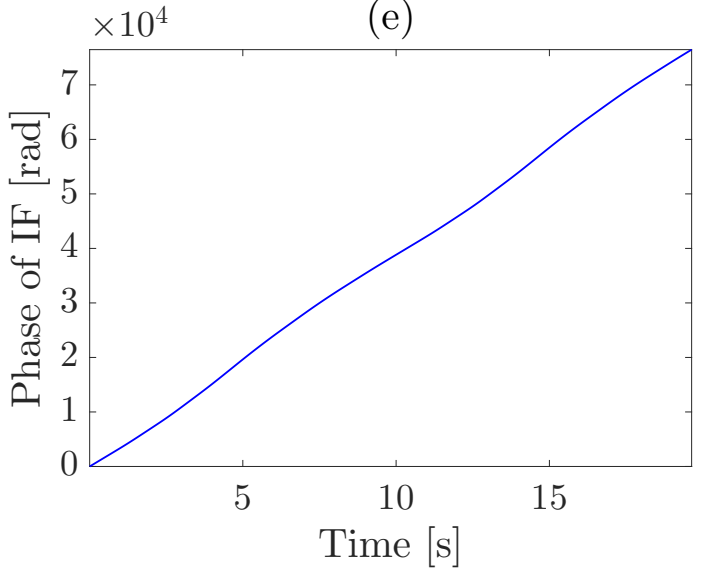

(b)

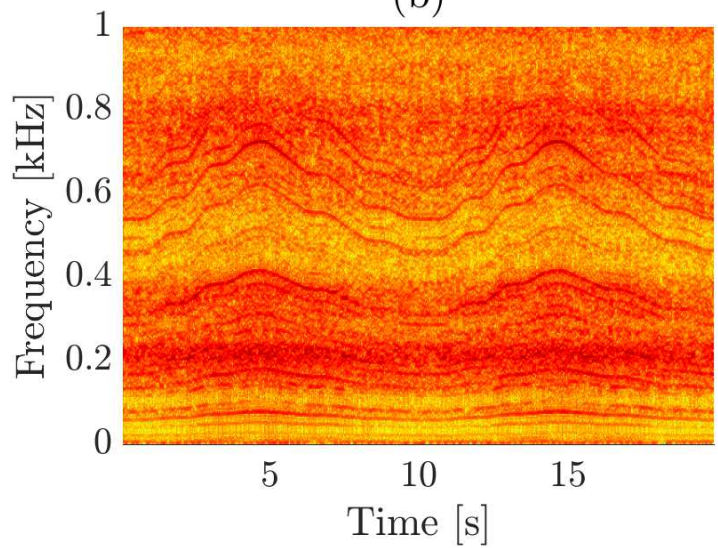

(d)

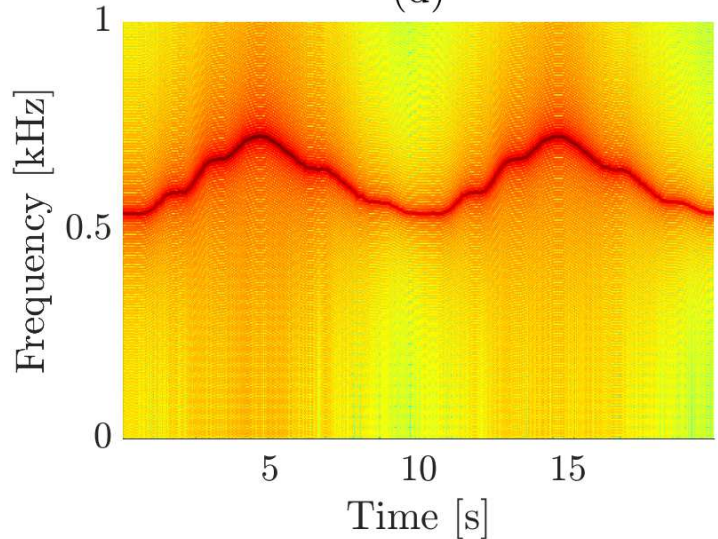

(f)

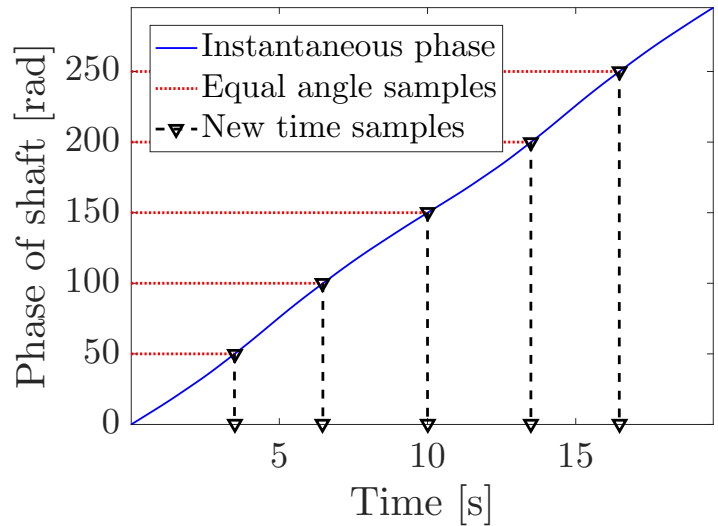

Figure 1: Key steps in the proposed tacholess order tracking method: (a) The acceleration signal and the rotational speed profile $\left(f_{r}\right)$ over time; (b) The spectrogram of the vibration signal; (c) The maxima tracked IF superimposed on the spectrogram in (b); (d) The spectrogram of the Vold-Kalman filtered signal using the maxima tracked IF in (c); (e) The phase of the IF; (f) The resampling process using the phase of the shaft.

mate the component of interest. Even though the spectrogram renders a limited time-frequency resolution, it is used for maxima tracking in this article and motivated by:

- It does not contain any interference or cross-terms such as the Wigner-Ville distribution $[18]$. 
- Fewer hyperparameters need to be optimised than for the Chirplet transform, which requires the chirp rate to be determined, and the continuous wavelet transform, which requires the scales as well as the wavelet basis function to be determined.

- It is fairly computationally efficient compared to the Chirplet transform.

The short-time Fourier transform (STFT), performed on a continuous signal $x(t)$ is in the form of,

$$
\Gamma(t, f)=\int_{-\infty}^{\infty} x(\tau) v(\tau-t) \exp (-2 \pi f j \tau) d \tau
$$

where a window function such as a Hamming window, is denoted by $v(t)$ and $j=\sqrt{-1}$. The spectrogram is related to the STFT in Equation (1) with $|\Gamma(t, f)|^{2}$. Equation (1) is easily implemented for discrete signals, since the fast Fourier transform is repeatedly applied on each windowed vibration signal segment to construct the discrete STFT and to obtain the discrete spectrogram. The discrete spectrogram is described by the integer variables $n$ and $l$ in this paper to obtain $|\Gamma[n, l]|^{2}$, where $t=n \Delta t_{\Gamma}$ seconds and $f=l \Delta f_{\Gamma}$ hertz. The time between horizontally adjacent windows in the spectrogram is denoted by $\Delta t_{\Gamma}$ and the frequency between vertically adjacent windows is denoted by $\Delta f_{\Gamma}$ and they describe the time and frequency resolution of the spectrogram, respectively.

A discrete signal evaluated at time increment $n$ is denoted by $x[n]$, where a continuous signal or a function is denoted by $x(t)$ in this article. The relationship between the discrete time increment $n$ and the continuous time $t$ is,

$$
t=n \Delta t
$$

where $\Delta t$ is the sampling period. Please note that all discrete indices, such as $n$ in $x[n]$ is in the range of $\left[0, N_{x}-1\right]$, if the number of indices in $x$ is $N_{x}$ for example.

\subsection{Proposed maxima tracking procedure}

Maxima tracking is performed to find, isolate and track an IF, denoted by $f_{\mathrm{IF}}$,

$$
f_{\mathrm{IF}}(t)=k \cdot c_{s} \cdot f_{r}(t)
$$


in a time-frequency distribution. The IF is related to the rotating frequency or speed of the shaft, denoted by $f_{r}(t)$, with the time-invariant factor $k \cdot c_{s}$. This indicates that it is the $k$ th harmonic of a component with a frequency of $c_{s} \cdot f_{r}(t)$, such as a gear mesh frequency or another shaft in the system. This IF presents itself as a ridge or a local maximum in the discrete time-frequency distribution, where the IF is estimated by solving a constrained optimisation problem in the standard form of,

$$
\begin{array}{ll}
\underset{l}{\operatorname{minimise}} & -|\Gamma[n, l]|^{2} \\
\text { subject to } & \left(l \cdot \Delta f_{\Gamma}-f_{c}[n]\right)^{2} \leq \Delta f_{c}^{2}
\end{array}
$$

where $f_{c}$ denotes the centre of the constraint, which is the predicted value of the IF at time increment $n ; \Delta f_{c}$ denotes the feasible bandwidth enforced by the constraint; and $l \cdot \Delta f_{\Gamma}$ is the current frequency that is investigated in the spectrogram in Hertz. If maxima tracking is performed as an unconstrained optimisation problem, different components will be tracked which results in erroneous phase estimation and order tracking results. The solution of Equation (4) at time increment $n$, denoted by $f_{\max }[n]$, is assumed to be related to the actual IF by,

$$
f_{\mathrm{IF}}\left(n \Delta t_{\Gamma}\right) \approx f_{\max }[n]
$$

where $\Delta t_{\Gamma}$ is the time difference between horizontally adjacent window centres in the spectrogram. Note that the noise and the fixed time-frequency resolution can result in some differences between the estimated IF $f_{\max }[n]$ and the actual IF $f_{\mathrm{IF}}$, which motivate the approximation used in Equation (5). Urbanek et al. [10] centred the constraint in Equation (4) about the maxima tracked frequency estimated at the previous time increment,

$$
f_{c}[n]=f_{\max }[n-1]
$$

with the frequency gradient i.e. acceleration information not taken into account. The feasible region (represented by $\Delta f_{c}$ ) must be increased to accommodate the errors made by Equation (6) when the rotating machine operates under large angular accelerations. However, if a large $\Delta f_{c}$ is used in the maxima tracking process, it is more susceptible to tracking incorrect frequency components such as other harmonics and background noise which are present in the large feasible region. 
Higher order frequency information needs to be incorporated into the maxima tracking process to make it more robust to track the IF in the presence of large accelerations. The Taylor series expansion of the IF in the signal about the previous time step,

$$
f_{\mathrm{IF}}(t)=f_{\mathrm{IF}}\left(t-\Delta t_{\Gamma}\right)+\Delta t_{\Gamma} \frac{d}{d t} f_{\mathrm{IF}}\left(t-\Delta t_{\Gamma}\right)+\frac{1}{2} \Delta t_{\Gamma}^{2} \frac{d^{2}}{d t^{2}} f_{\mathrm{IF}}\left(t-\Delta t_{\Gamma}\right)+\ldots
$$

can be used to predict the value of the IF at the current time step, denoted by $t=n \Delta t_{\Gamma}$, if all of the derivatives of $f_{\mathrm{IF}}$ are continuous. This prediction is used to centre the constraint,

$$
f_{c}[n]=f_{\mathrm{IF}}\left(n \Delta t_{\Gamma}\right)
$$

used in Equation (4). Equation (6) can be obtained from Equation (7) and Equation (8) by neglecting the higher order information i.e. no acceleration is present and by assuming Equation (5) is valid. The problem with Equation (7) is that the gradients of the IF are unknown and the estimated value of the IF at the previous time increment $f_{\max }[n-1]$ is known instead of the actual value $f_{\mathrm{IF}}\left(t-\Delta t_{\Gamma}\right)$. The spectrogram contains discrete time steps and therefore the gradients in Equation (7) can only be estimated by using finite difference schemes on the previous

IF estimates i.e. $f_{\max }[n-1], f_{\max }[n-2]$ etc. The estimate of the IF contains noise due to ridge smearing and background noise in the spectrogram, which makes finite difference schemes poor approximations to the gradients in Equation (7).

A probabilistic approach is proposed in this article to infer the actual IF so that higher order information can be incorporated into the maxima tracking process. It is assumed that the true $\mathrm{IF}, f_{\mathrm{IF}}$, is related to its representation in the spectrogram or its estimate from the spectrogram, $f_{\text {max }}$, with,

$$
f_{\max }[n]=f_{\mathrm{IF}}\left(n \Delta t_{\Gamma}\right)+\nu
$$

where $\nu$ is a sample from a zero mean Gaussian with variance $\sigma^{2}$, which represents the noise that is attributed to ridge smearing and other noise components in the spectrogram. It is assumed that the noise in the spectrogram is Gaussian distributed and therefore the estimated IF (or maxima tracked frequency) and the true IF is related with the Gaussian probability density 
function $p$ given by,

$$
p\left(f_{\max }[n] \mid f_{\mathrm{IF}}\left(n \Delta t_{\Gamma}\right), \sigma^{2}\right)=\frac{1}{\sqrt{2 \sigma^{2} \pi}} \exp \left(-\frac{\left(f_{\max }[n]-f_{\mathrm{IF}}\left(n \Delta t_{\Gamma}\right)\right)^{2}}{2 \sigma^{2}}\right)
$$

where the true IF, denoted by $f_{\mathrm{IF}}$, remains unknown. A model of the true IF, denoted by $f_{F}$ and represented by a $N_{p}$-order polynomial in the form of,

$$
f_{F}(t)=w_{0}+w_{1} t^{1} \ldots w_{N_{p}} t^{N_{p}}
$$

is used instead of the unknown IF $f_{\mathrm{IF}}$. Table 1 summarises the difference between the different notations for the IF. The polynomial weights $\boldsymbol{w}=\left[w_{0}, w_{1}, \ldots w_{N_{p}}\right]^{T}$ are estimated by performing Table 1: The differences between $f_{\max }, f_{\mathrm{IF}}$ and $f_{F}$.

$f_{\mathrm{IF}}(t) \quad$ Is the true, continuous IF that need to be estimated by the process.

$f_{\max }[n]$ Is the IF that is inferred at discrete time steps from the maxima tracking process.

$f_{F}(t) \quad$ Is a model that captures the local gradient characteristics of the estimated IF.

maximum likelihood,

$$
\hat{\boldsymbol{w}}=\underset{\boldsymbol{w}}{\operatorname{argmax}} \prod_{i=n-N_{m}}^{n-1} p\left(f_{\max }[i] \mid f_{F}\left(i \Delta t_{\Gamma}\right), \sigma^{2}\right)
$$

over the previous $N_{m}$ time steps by assuming that the data are identically and independently distributed. The resulting weights [19],

$$
\hat{\boldsymbol{w}}=\left(\boldsymbol{Q}^{T} \boldsymbol{Q}\right)^{-1} \boldsymbol{Q}^{T} \boldsymbol{f}_{\max }
$$

of the maximum likelihood solution are computed from the $N_{m} \times\left(N_{p}+1\right)$ design matrix $\boldsymbol{Q}$ and the previous $N_{m}$ estimates of the IF, denoted by a $N_{m} \times 1$ vector $\boldsymbol{f}_{\text {max }}$. The design matrix of the $N_{p}$-order polynomial, in the form of,

$$
\boldsymbol{Q}=\left[\begin{array}{cccc}
1 & \Delta t_{\Gamma} \cdot(n-1) & \ldots & \left(\Delta t_{\Gamma} \cdot(n-1)\right)^{N_{p}} \\
1 & \Delta t_{\Gamma} \cdot(n-2) & \ldots & \left(\Delta t_{\Gamma} \cdot(n-2)\right)^{N_{p}} \\
\vdots & \vdots & \ddots & \vdots \\
1 & \Delta t_{\Gamma} \cdot\left(n-N_{m}\right) & \ldots & \left(\Delta t_{\Gamma} \cdot\left(n-N_{m}\right)\right)^{N_{p}}
\end{array}\right]
$$


and the vector of the previous $N_{m}$ estimates of the IF,

$$
\boldsymbol{f}_{\max }=\left[\begin{array}{c}
f_{\max }[n-1] \\
f_{\max }[n-2] \\
\vdots \\
f_{\max }\left[n-N_{m}\right]
\end{array}\right]
$$

are used in Equation (13) to obtain the weights used in Equation (11). The polynomial function in Equation (11) satisfies the Taylor series expansion of the IF in Equation (7), which means that if a first order polynomial function is used, then the assumption is made that the angular acceleration is constant.

It is strongly suggested to use a first order polynomial to ensure that only a small number of training points $\left(N_{m}\right)$ are required to avoid overfitting, solving Equation (13) repeatedly remains computationally efficient and that errors in the extrapolation process are minimised. The number of training points are $N_{m}=5$ in this article, which makes the first order approximation sensible for $\Delta t_{\Gamma}$, which was in the range of $[0.028,0.05]$ seconds for all spectrograms in this article. By using Equation (6), the IF estimate at the first time increment in the maxima tracking process needs to be provided by the user. If the constraint in Equation (4) is centred by the prediction made by Equation (11) from a first order polynomial, then the initial gradient needs to be provided as well. To make this process easier, it is assumed that the initial gradient is zero, which essentially results in Equation (6) to be used for the initial steps. This assumption is reasonable if other frequency components are not in close proximity to the IF under consideration, otherwise a rough estimate of the gradient can be provided as input if the aforementioned assumption leads to incorrect results. In this study the first three estimates of the IF are obtained by using Equation (4) with Equation (6) and thereafter Equation (11) can be used to estimate $f_{c}$. The initial estimate of the IF is obtained by inspecting the spectrogram and $c_{s} \cdot k$, in Equation (3) and is estimated by roughly knowing the operating range of the machine or using a simple device such as a hand-held tachometer. 
Since it is a constrained minimisation problem, the Lagrangian in the form of,

$$
\begin{aligned}
\mathcal{L}_{n}(l, \boldsymbol{\lambda}, \boldsymbol{s})=-|\Gamma[n, l]|^{2} & +\lambda_{1}\left(\left(l \Delta f_{\Gamma}-f_{\max }[n]\right)^{2}-\Delta f_{c 1}^{2}+s_{1}^{2}\right) \\
& +\lambda_{2}\left(\left(l \Delta f_{\Gamma}-f_{F}\left(n \Delta t_{\Gamma}\right)\right)^{2}-\Delta f_{\mathrm{c} 2}^{2}+s_{2}^{2}\right)
\end{aligned}
$$

is minimised to find the frequency $f=l \Delta f_{\Gamma}$ that minimises the cost function $-|\Gamma[n, l]|^{2}$ and adheres to the constraints. The Lagrangian multiplier and slack variable associated with the $i$ th constraint are denoted by $\lambda_{i}$ and $s_{i}$, respectively. Equation (16) can be minimised by solving a set of linear or quadratic programming sub-problems, by solving a penalised unconstrained cost function etc. [20]. The penalised unconstrained cost function in the form of,

$$
\begin{aligned}
\kappa(l, \boldsymbol{\rho}, n)=-|\Gamma[n, l]|^{2} & +\rho_{1} \max \left[0,\left(l \Delta f_{\Gamma}-f_{\max }[n-1]\right)^{2}-\Delta f_{c 1}^{2}\right] \\
& +\rho_{2} \max \left[0,\left(l \Delta f_{\Gamma}-f_{F}\left(n \Delta t_{\Gamma}\right)\right)^{2}-\Delta f_{c 2}^{2}\right]
\end{aligned}
$$

is minimised using a brute force approach, since the limited number of feasible solutions at each time step, due to the discrete spectrogram, makes it easy to obtain a minimum. The bandwidth describing the feasible region enforced by the two constraints are denoted by $\Delta f_{c 1}$ and $\Delta f_{c 2}$, respectively. The parameters $\rho_{1}$ and $\rho_{2}$ must be chosen so that the cost function in Equation (17) is dominated by the constraint terms if the constraints are violated. A procedure is proposed in section 2.5 to choose the values of $\rho_{1}$ and $\rho_{2}$. In this paper the feasible bandwidths $\left(\Delta f_{c 1}\right.$ and $\left.\Delta f_{c 2}\right)$ in Equation (17) have the same value. The components of the vector $\boldsymbol{\rho}=\left[\rho_{1}, \rho_{2}\right]$, in Equation (17), have the following values,

$$
\rho_{1}= \begin{cases}10^{9} & \text { if } n=0,1,2 \\ 0 & \text { otherwise }\end{cases}
$$

and,

$$
\rho_{2}= \begin{cases}10^{9} & \text { if } n>2 \\ 0 & \text { otherwise }\end{cases}
$$

where only one of the constraints are active at a time due to the choice of $\boldsymbol{\rho}$. The estimate of 
the instantaneous frequency is obtained from,

$$
f_{\max }[n]=\Delta f_{\Gamma} \cdot \underset{l}{\operatorname{argmin}} \kappa(l, \boldsymbol{\rho}, n)
$$

where $\kappa(l, \boldsymbol{\rho}, n)$ is given by Equation (17). Note that the solution of Equation (17) is also the solution of Equation (4) if both constraints are incorporated into Equation (4) similarly to Equation (17). The maxima tracking process is illustrated in Figure 2. The method implemented by Urbanek et al. [10] essentially uses $\rho_{1}>0$, while $\rho_{2}$ is always zero in Equation (17). In this

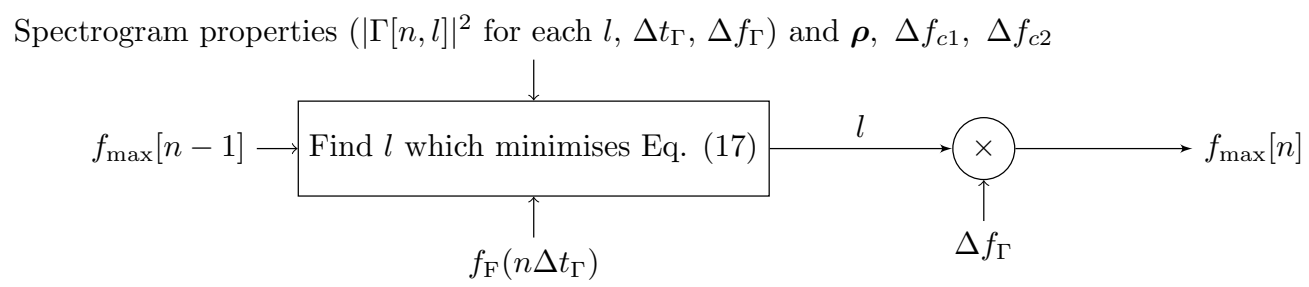

Figure 2: The maxima tracking process is illustrated in this Figure at time increment $n$ and this is performed for each time increment $n$. Note that for example if $\rho_{1}$ in Equation (17) is zero, then $f_{\max }[n-1]$ do not need to be used in the process.

article the proposed maxima tracking algorithm is compared to the method by Urbanek et al. [10] to highlight the benefits of including acceleration information into the maxima tracking process.

\subsection{The Vold-Kalman filter}

The Vold-Kalman filter (VKF) is a bandpass filter with a varying centre frequency and bandwidth. If the centre frequency of the constraint in Equation (4) is set equal to the estimated IF, denoted by $\boldsymbol{f}_{\max }$, and the bandwidth is set sufficiently fine, then a mono-component signal, denoted by $\mathbf{x}_{\mathrm{IF}}$ can be extracted from a multicomponent vibration signal $\boldsymbol{x}$. Note that a discrete signal is investigated in this section and therefore the whole signal is denoted by $\boldsymbol{x}$ i.e. a onedimensional vector over all time steps. The signal at increment $n$ is denoted by $x[n]$. The Vold-Kalman filter allows slight errors in the maxima tracking process and this is the reason why a Vold-Kalman filter is used, instead of integrating the estimated maxima tracked frequency, obtained from Equation (20). 
Two generations of the VKF exist, namely the angular-velocity (or first generation) and the angular-displacement (or second generation) VKF. The angular-velocity VKF aims to estimate the mono-component signal, while the angular-displacement VKF aims to estimate the envelope of the mono-component signal with the supplied frequency content [21]. The signal containing the IF that needs to be extracted, $x_{\mathrm{IF}}[n]$ is related to the vibration signal $x[n]$ in the data equation of the VKF,

$$
x[n]=x_{\mathrm{IF}}[n]+\zeta[n],
$$

where $\zeta[n]$ represents the other sinusoidal components and the background noise at time $t=n / f_{s}$. The structural equation for the angular-velocity VKF,

$$
x_{\mathrm{IF}}[n]-2 \cos \left(2 \pi f_{\mathrm{IF}}[n] f_{s}^{-1}\right) x_{\mathrm{IF}}[n+1]+x_{\mathrm{IF}}[n+2]=\varepsilon[n]
$$

and of the angular-displacement VKF,

$$
\beta[n]-\beta[n+1]=\varepsilon[n]
$$

describes the characteristic of interest (either the signal component or its envelope) and $\varepsilon[n]$ is known as the non-homogeneity term at time increment $n$ which represents the error in the left-side of the structural equation. Equation (23) is for a one-pole angular-displacement filter, where the structural equation for other poles are found in the paper by Tuma [21]. The envelope $\beta[n]$ is related to the signal of interest by,

$$
x_{\mathrm{IF}}[n]=\beta[n] \exp \left(\frac{2 \pi j}{f_{s}} \sum_{i=0}^{n} f_{\mathrm{IF}}[i]\right)
$$

where $f_{s}$ is the sampling frequency of the signal $x_{\mathrm{IF}}, j=\sqrt{-1}$ and the instantaneous frequency $f_{\mathrm{IF}}[i]$ is obtained from Equation (5) and by using the maxima tracking information. There are three sets of unknowns in Equation (21), (22) and (23) namely $\boldsymbol{\varepsilon}, \boldsymbol{\zeta}$ and either $\boldsymbol{\beta}$ for the angulardisplacement VKF or $\boldsymbol{x}_{\mathrm{IF}}$ for the angular-velocity VKF. The dimensions of the aforementioned vectors are $N_{x} \times 1$, where $N_{x}$ is the number of samples in the signal $\boldsymbol{x}$. This results in an underdetermined system of equations, which is solved by finding the characteristic of interest $\left(\boldsymbol{x}_{\mathrm{IF}}\right.$ or $\left.\boldsymbol{\beta}\right)$ that simultaneously minimises $\boldsymbol{\varepsilon}$ and $\boldsymbol{\zeta}$. This is achieved by solving the multi-objective 
optimisation problem with the weighted sum method in the form of [21],

$$
\kappa=a^{2} \varepsilon^{T} \varepsilon+\zeta^{T} \zeta
$$

where $a$ is the weighting factor of the Vold-Kalman filter and it describes the relative importance of minimising $\varepsilon^{T} \boldsymbol{\varepsilon}$ instead of $\boldsymbol{\zeta}^{T} \boldsymbol{\zeta}$. The weighting factor is given by [21],

$$
a=\sqrt{\frac{\sqrt{2}-1}{2\left(1-\cos \left(2 \pi f_{b} / f_{s}\right)\right)}}
$$

for a one-pole angular-displacement VKF filter, where $f_{b}$ is the bandwidth of the filter. A unique weighting factor, found in Equation (26), can be applied at each time step, which provides more flexibility to the VKF,

$$
\kappa=\varepsilon^{T} \boldsymbol{A}^{T} \boldsymbol{A} \boldsymbol{\varepsilon}+\boldsymbol{\zeta}^{T} \boldsymbol{\zeta}
$$

where the $N_{x} \times N_{x}$ weight matrix $\boldsymbol{A}$ is a diagonal matrix containing the local weight factors and $N_{x}$ is the number of samples in the signal $\boldsymbol{x}$. The component at time increment $n$, denoted by $A[n, n]$, describes the importance of minimising $\varepsilon^{T} \varepsilon$ instead of $\boldsymbol{\zeta}^{T} \boldsymbol{\zeta}$ at the time increment. The choice of weighting factor at time increment $n$, denoted by $A[n, n]$, determines the rise time as well as the bandwidth of the filter and is very important to the success of the VKF [22]. The angular-displacement VKF allows multiple components to be tracked, while the angular-velocity VKF is constrained to a single component [21]. Due to the nature of the data and structural equations, the optimal solution of the cost function, where the cost function is given by Equation (27), can be obtained in closed form if only a single component is tracked [21].

Pan and Lin [22] found that the angular-displacement VKF is able to estimate the component of interest accurately in the presence of crossing orders. It obtains a better rise time and bandwidth combination than the angular-velocity VKF as well. The angular-displacement VKF does not have any frequency nor slew rate limitations [23]. In the authors' experience, it is found that the one-pole angular-displacement filter is able to extract the component of interest using a small relative bandwidth, given by,

$$
B_{w}=\frac{f_{b}[n]}{f_{\mathrm{IF}}[n]}
$$

without any numerical problems. A one-pole angular-displacement VKF used with a small 
relative bandwidth makes the phase error independent of the harmonic that is filtered i.e. $k$ in Equation (3), which reduces the number of hyperparameters that need to be optimised. Hence a one-pole angular-displacement VKF is used in this study to obtain a mono-component signal from the multicomponent vibration signal. If $B_{w}$ is large, it means that relatively large maxima tracking errors are accommodated in the process at the risk of other frequency components being passed through the passband as well. The values of $B_{w}$ are provided in section 3 for each investigation.

\subsection{Instantaneous phase estimation and resampling}

The phase estimation process is presented in this section for continuous signals, however it is easily extended to discrete signals obtained from the maxima tracking and the Vold-Kalman filtering processes. The mono-component signal obtained from the VKF in the previous section, denoted by $x_{\mathrm{IF}}$, is transformed with the Hilbert transform,

$$
H\left(x_{\mathrm{IF}}(t)\right)=\frac{1}{\pi} \int_{-\infty}^{\infty} x_{\mathrm{IF}}(\tau) \frac{1}{t-\tau} d \tau
$$

and used to obtain the instantaneous phase of this signal,

$$
\phi_{\mathrm{IF}}(t)=\arctan \left(\frac{H\left(x_{\mathrm{IF}}(t)\right)}{x_{\mathrm{IF}}(t)}\right)
$$

which is constrained to $[-\pi, \pi]$ radians due to the arctangent function. This phase, obtained from Equation (30), is unwrapped to obtain the cumulative phase of the estimated IF component over time. The tracked IF component is related to the rotating shaft of interest with Equation (3) if Equation (5) is valid. The aforementioned relationship is used to obtain the instantaneous phase of the shaft,

$$
\phi_{r}(t)=\frac{1}{k c_{s}} \operatorname{unwrap}\left(\phi_{\mathrm{IF}}(t)\right)
$$

at time step $t$. The instantaneous phase of the shaft over time, obtained from Equation (31), is used to resample the signal into equal angular increments as shown by (f) in Figure 1.

\subsection{Guidelines for choosing parameters}

The adjustable parameters in the proposed tacholess order tracking process are the 
- properties of the spectrogram namely, the window length $\left(L_{w}\right)$, window overlap $\left(L_{o}\right)$, window type and zero padding $\left(L_{z}\right)$; where $L_{w}, L_{o}$ and $L_{z}$ are given in the number of samples that is used.

- initial value of the estimated instantaneous frequency $f_{\max }$ and its relationship with the rotational speed of the shaft $c_{s} \cdot k$.

- properties of the maxima tracking process such as $N_{m}$ and $N_{p}$.

- allowable bandwidth of the constraints of the maxima tracking process $\Delta f_{c}$ and $\rho$.

- relative bandwidth, type and order of the Vold-Kalman filter.

Some guidelines are proposed in this section to select the unknown parameters. Please note that the maxima tracking properties such as $N_{m}$ and $N_{p}$ are discussed and motivated in section 2.2 while the Vold-Kalman properties are thoroughly discussed and motivated in section 2.3 and are therefore not repeated in this section. The aforementioned parameters are summarised in section 3.2.3 for each investigated numerical and experimental case.

The time and frequency resolution of the spectrogram is related to the window length, window overlap and zero padding by $\Delta t_{\Gamma} \propto L_{w}-L_{o}$ and $\Delta f_{\Gamma} \propto 1 /\left(L_{w}+L_{z}\right)$, respectively. The appropriate window length is problem dependent and should be chosen to have sufficient time resolution to estimate varying rotational speeds without a significant loss in frequency resolution. In this paper, typical window lengths of $0.1-0.5$ seconds are investigated. Large window overlaps are suggested (typically $50 \%-90 \%$ of $\left.L_{w}\right)$ and zero padding of $L_{z}=2 \cdot\left(2^{L L_{w}}-L_{w}\right)$ where $L L_{w}=$ ceil $\left(\log _{2}\left(L_{w}\right)\right)$ to ensure that relatively good time and frequency resolutions are obtained. The ceiling function is denoted by ceil(). Rectangular windows are used in this paper similar to [9], but other windows can be investigated if desired.

The initial value of $f_{\max }$ can be chosen by inspecting the spectrogram and looking for dominant meshing components, while $c_{s}$ will be the number of teeth on the gear that is connected to the shaft under consideration. The constant $k$ can be estimated by approximately knowing the speed of the shaft under consideration. Hand-held tachometers can for example be used to obtain a rough estimate of the operating range of the shaft under consideration, which can subsequently be used to estimate $k$. 
The feasible bandwidth $\Delta f_{c}$ indicates the allowable error when centring the constraint $f_{c}$ and the appropriate characteristics are dependent on the harmonic that is tracked as well as the local frequency and noise characteristics in the spectrogram. Hence, in many cases the appropriate $\Delta f_{c}$ is chosen on a trail and error basis. Due to the fact that a first order polynomial is used, some errors can be present in the prediction due to unpredictable changes in operating conditions and therefore using $\Delta f_{c}=0$ is unrealistic. It is suggested to start with $\Delta f_{c}$ in the range of $5-20 \mathrm{~Hz}$ for the datasets that are investigated.

The parameter $\rho$ ensures that the constraint violation dominate the cost function in Equation (17). Assume that if constraint $i$, governed by $\rho_{i}$, is violated by $(q-1) \Delta f_{c}$ where $q>1$, then the objective function $\kappa(l, \boldsymbol{\rho}, n)>0$ for all integers $l$ and $n$. This condition will guarantee that $f_{\max }$, obtained from the optimisation process, will be within the range $\left[f_{c}-q \Delta f_{c}, f_{c}+q \Delta f_{c}\right]$ for

all time increments. The solution to this problem is $\rho_{i}>\left|\Gamma_{\max }\right|^{2} /\left(\left(q^{2}-1\right) \Delta f_{c}^{2}\right)$ where $\left|\Gamma_{\max }\right|^{2}$ is the absolute maximum value of the spectrogram and $q$ can for example be 1.0001 to simulate a $0.01 \%$ constraint violation.

\section{Validation}

Three numerical and three experimental datasets are used to validate the proposed tacholess order tracking method. The performance of the proposed maxima tracking algorithm is critically compared to the performance of the maxima tracking method used by [10], where the latter is used as a benchmark. After the maxima tracking process is completed, the same process (i.e. the Vold-Kalman filter, Hilbert transform etc.) is followed for both maxima tracking algorithms. The results from the benchmark maxima tracking algorithm is indicated by (a) and the proposed method is indicated by (b) in all subsequent figures.

The vibration signal is order tracked using the estimated instantaneous phase and therefore the performance of the tacholess order tracking method (or the maxima tracking algorithm) is quantified using the estimated phase error. The phase error, in this study, is computed from,

$$
e(t)=\left|\phi_{r}(t)-\phi_{a}(t)\right|
$$

where the cumulative analytical and estimated phase of the shaft, in radians, are denoted by $\phi_{a}$ 
and $\phi_{r}$ respectively. The maximum relative phase error is computed from,

$$
e_{R}=\frac{\max (e(t))}{\max \left(\phi_{a}(t)\right)}
$$

where $\max \left(\phi_{a}(t)\right)$ denotes the maximum value of the analytical instantaneous phase for the time interval and $e(t)$ is calculated from Equation (32). The numerical data are considered first in this section, whereafter the experimental data are presented. Note that the parameters that are used in the tacholess order tracking process are summarised for the numerical and experimental data in section 3.2.3.

\subsection{Numerical data}

Two rotational speed profiles are investigated on the numerical data. The first rotational speed profile of the shaft of interest in Hertz,

$$
f_{r, 1}(t)=200 t+50
$$

has a constant angular acceleration of $400 \pi\left[\mathrm{rad} / \mathrm{s}^{2}\right]$ over 20 seconds. The second rotational speed profile in Hertz,

$$
f_{r, 2}(t)=\frac{1}{5} \cos (0.1 \pi t)+\frac{1.25}{\pi}
$$

has a varying angular acceleration. Note that the rotational speed in Equation (35) is very small, but a gear mesh frequency of a simulated gearbox, given by $f_{r, 2} \cdot 40$ is introduced and tracked on the spectrogram of the simulated signal, which has a significantly larger frequency gradient.

The first simulated vibration signal is in the form of,

$$
x_{1}(t)=\sin \left(2 \pi \int_{0}^{t} f_{r, 1}(\tau) d \tau\right)
$$

and contains a frequency component given by Equation (34). Note that the signal is a monocomponent signal and therefore the Hilbert transform can directly be applied. However, this signal is investigated to evaluate the performance of the proposed method on a mono-component dataset and then an additional component is incorporated in the second simulated vibration signal. 
The second simulated vibration signal is in the form of,

$$
x_{2}(t)=\sin \left(2 \pi \int_{0}^{t} f_{r, 1}(\tau) d \tau\right)+\sin \left(2 \pi 10^{3} t\right)
$$

where it contains the rotational speed given in Equation (34) and an additional constant frequency component. This rotational speed invariant component simulates noise generated by resonance bands or by environmental effects. The effect of adding spurious components to the signal is investigated by comparing the results of the maxima tracking algorithms on the signal given by Equation (36) and Equation (37). The third and last simulated vibration signal is in the form of,

$$
\begin{aligned}
x_{3}(t)=\sum_{s=1}^{3} & {\left[1.5 \sin \left(2.0 \pi s \int_{0}^{t} f_{r, 2}(\tau) d \tau\right)+\sin \left(80.0 \pi s \int_{0}^{t} f_{r, 2}(\tau) d \tau\right)\right]+} \\
& 0.5 \sin (2 \pi[1.65 t+2.0] t)+0.5 \sin (80.0 \pi t)
\end{aligned}
$$

and contains a shaft component given by Equation (35) and its three harmonics, a gear mesh frequency $\left(40 \cdot f_{r, 2} \mathrm{~Hz}\right)$ and its three harmonics, a linear component and a constant frequency component. The two latter frequency components are independent of the rotation speed and complicates the maxima tracking task.

The ratio of the $\Delta f_{c}$ to spectrogram frequency resolution, is selected as 16 for the first two simulated signals and 2.73 for the last signal for the maxima tracking process. Note that it should be significantly larger for the first two simulated signals due to the large frequency gradient. The VKF relative bandwidth is selected to be 0.1 for the first two signals and $10^{-4}$ for the last signal. Gaussian noise with a standard deviation of 1.0 is added to the first two signals and Gaussian noise with a standard deviation of 2.5 is added to the last simulated signal.

The spectrogram of the signal given by Equation (36) is computed and the maxima tracking algorithms are performed on the spectrogram, separately. The result of the benchmark and the proposed maxima tracking procedures are superimposed on the spectrogram in Figure $3 \mathrm{i}$ and indicated by (a) and (b), respectively. The angular-displacement VKF is applied to the signal, whereafter the Hilbert transform is applied to calculate the phase of the component. The phase error is calculated with Equation (32) and given in Figure 3ii. Both the maxima tracking approaches resulted in very similar phase errors, with the proposed maxima tracking 


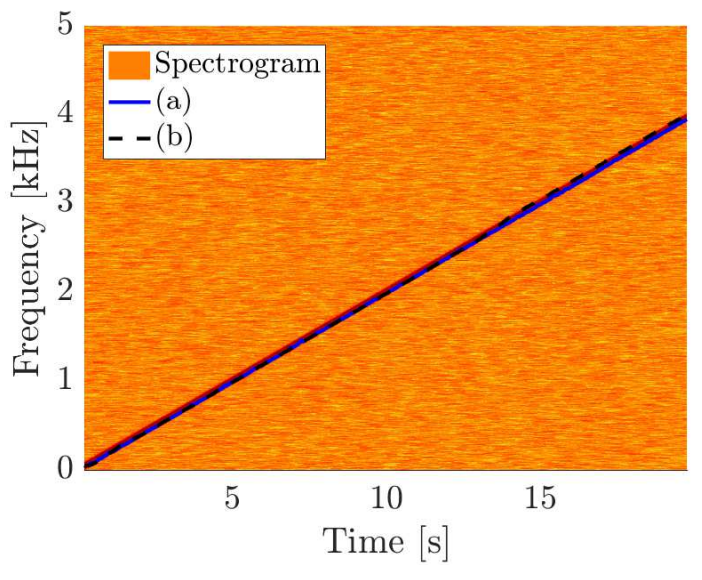

(i)

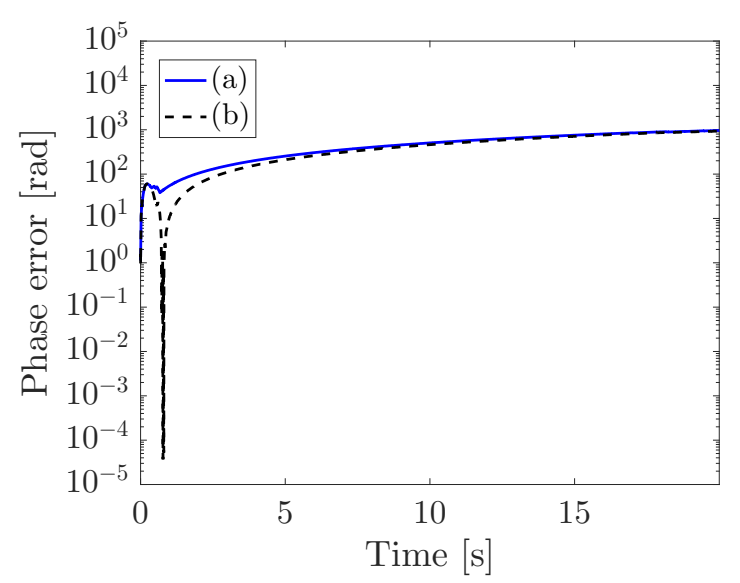

(ii)

Figure 3: The result of the proposed tacholess order tracking method on the first simulated signal, given by Equation (36). The maxima tracking results are superimposed on the spectrogram of the signal in Figure 3i with the resulting phase errors, calculated from Equation (32), shown in Figure 3ii. The benchmark maxima tracking algorithm is indicated as (a) in the figures and the proposed maxima tracking algorithm is indicated by (b).

method performing slightly better. Note that the phase error resulted from a constant frequency error which results from the limited time-frequency resolution of the spectrogram and the large frequency gradient. The constant frequency errors can be reduced by increasing the relative relative bandwidth of the VKF. However care must be taken in multicomponent signals where there is a risk of tracking another frequency component. Even though there is a linear phase error, the final phase error is significantly smaller than the final instantaneous phase of 251324.899 radians which results in a maximum relative phase error, calculated by Equation (33), of 0.3849\% and $0.3724 \%$ for the benchmark and the proposed method, respectively.

The same process is followed for the second simulated vibration signal given in Equation (37). The results from the two maxima tracking methods are superimposed on the spectrogram of the vibration signal in Figure 4i. The signal contains two frequency components with equal amplitudes, where the one frequency component is unrelated to the rotational speed of the shaft of interest. The two components can only be distinguished from one another in the spectrogram by evaluating the frequency gradient. The results in Figure 4i indicate that the benchmark method follows the signal component with a gradient of zero, which is expected. In contrast, the proposed method tracks the correct frequency component. The phase errors are compared in Figure 4ii, which proves that the proposed method performs significantly better and it is significantly more robust on a signal with spurious components. The performance of the proposed maxima tracking 


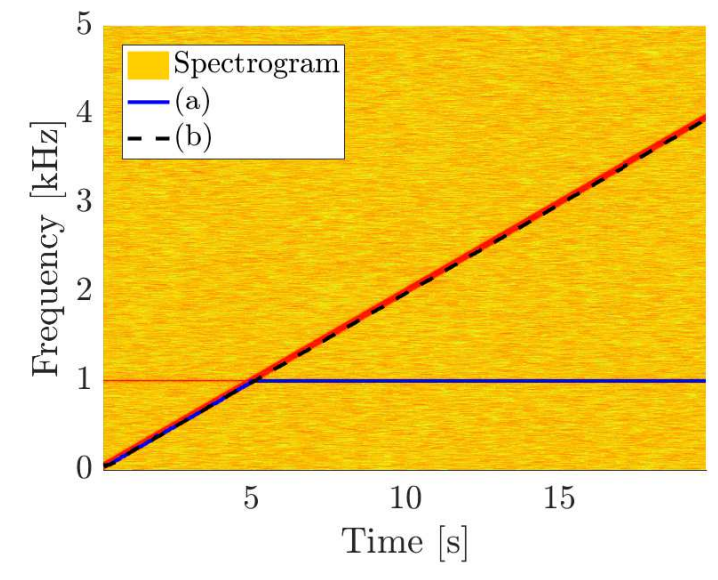

(i)

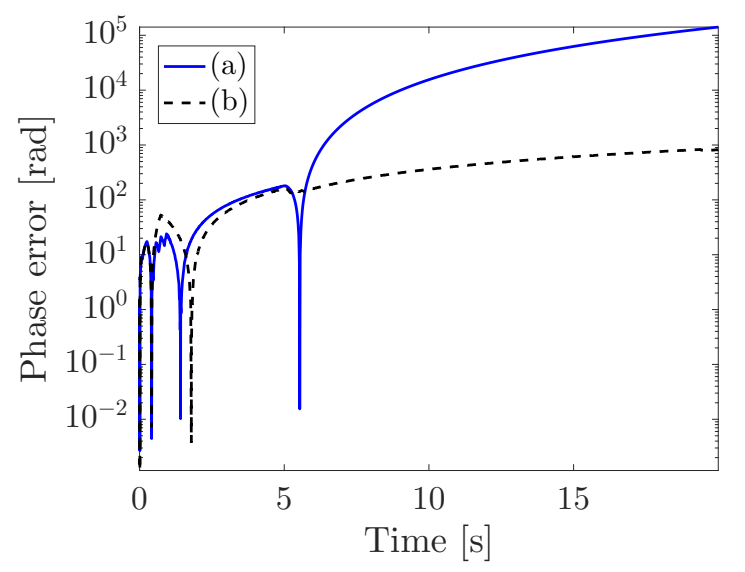

(ii)

Figure 4: The result of the proposed method on the second simulated signal, given by Equation (37). The maxima tracking results are superimposed on the spectrogram of the signal in Figure 4i with the resulting phase errors, calculated from Equation (32), shown in Figure 4ii.

algorithm does not change significantly between signal 1 and signal 2 (i.e. the addition of a spurious component), however the performance of the benchmark method is sensitive to the addition of another component.

The last simulated signal, given in Equation (38), is investigated similarly to the previous methods. The resulting maxima tracking results are superimposed in Figure 5i on the spectrogram and result in the phase errors given in Figure 5ii. A similar result is observed in Figure

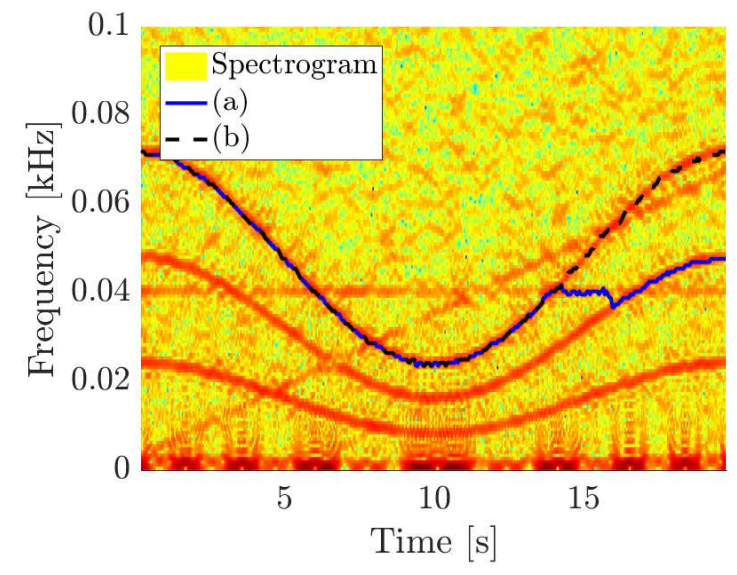

(i)

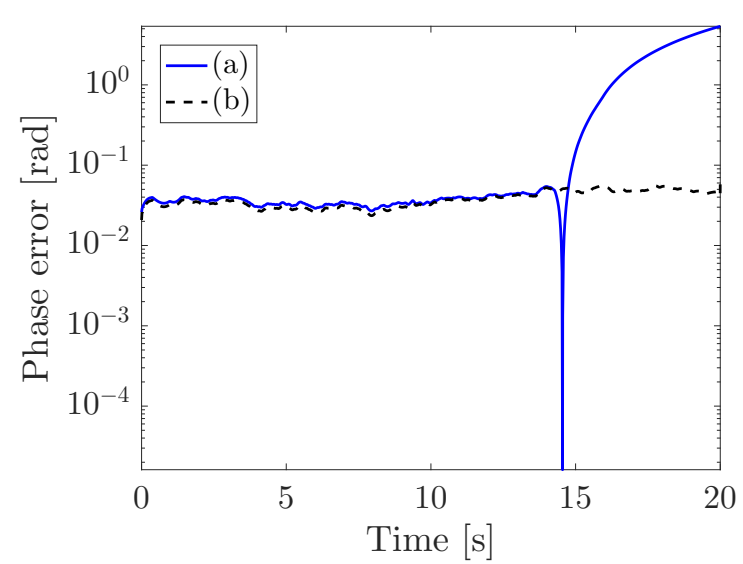

(ii)

Figure 5: The result of the proposed method on the third simulated signal, given by Equation (38). The maxima tracking results are superimposed on the spectrogram of the signal in Figure 5i with the resulting phase errors, calculated from Equation (32), shown in Figure 5ii.

5 as in Figure 4. The benchmark maxima tracking algorithm is incapable of distinguishing between the constant frequency components and the IF that needs to be tracked in Figure 5i. The 
proposed maxima tracking algorithm tracks the correct IF for the whole duration of the signal. This is reflected in the phase errors compared in Figure 5ii.

The performance of the two maxima tracking approaches is summarised in Table 2. The roughly estimated signal-to-noise ratio in decibels $(\mathrm{db})$,

$$
\mathrm{SNR}_{\mathrm{db}}=10 \log 10\left(\frac{\sum_{i=0}^{N-1}\left(\left|\Gamma_{\mathrm{IF}}[i]\right|^{2}\right)^{2}}{\sum_{i=0}^{N-1}\left(\left|\Gamma_{\text {noise }}[i]\right|^{2}\right)^{2}}\right)
$$

is presented in the table as well, where $\left|\Gamma_{\mathrm{IF}}[i]\right|^{2}$ is the magnitude of the spectrogram of the IF at time step $i$ and $\left|\Gamma_{\text {noise }}[i]\right|^{2}$ presents the amplitude of the noise around the IF in the spectrogram at time step $i$. The local signal-to-noise ratio is presented to give the approximate conditions in which the maxima tracking was performed in. The proposed maxima tracking algorithm, Table 2: Summary of the maxima tracking results from the numerical signals. All the phase units are in radians, except the maximum relative phase error.

\begin{tabular}{lccc}
\hline & Signal 1 & Signal 2 & Signal 3 \\
\hline Maximum phase completed & 251324.899 & 251324.899 & 314.1593 \\
Signal-to-noise ratio (db) & 52.79 & 22.71 & 20.35 \\
Benchmark method [10]: & & & \\
$\quad$ RMS phase error & 576.3152 & 54636.0666 & 1.4576 \\
$\quad$ Maximum phase error & 967.3627 & 141189.2577 & 5.3663 \\
$\quad$ Maximum relative phase error (\%) & 0.38491 & 56.1780 & 1.70815 \\
Proposed method: & & & \\
$\quad$ RMS phase error & 540.9771 & 459.872 & 0.039169 \\
$\quad$ Maximum phase error & 935.9197 & 829.7571 & 0.063935 \\
$\quad$ Maximum relative phase error (\%) & 0.372394 & 0.330153 & 0.020351 \\
\hline
\end{tabular}

in contrast to the benchmark algorithm, is able to track the correct frequency component in the presence of other spurious components. The spurious noise components emulate resonance bands which are excited due to the presence of damage and other noise components during data acquisition, which is present when performing condition monitoring on rotating machines. The quality of the order tracked vibration signal depends on the accuracy of the estimated instantaneous phase and is investigated on the experimental data in the next section.

\subsection{Experimental data}

Three aspects of the proposed tacholess order tracking method is evaluated in this section: The ability of the proposed tacholess order tracking method,

1. to estimate the instantaneous phase correctly; 
2. to order track the vibration signal;

3. to estimate the rotational speed of the shaft of interest.

\subsubsection{Experimental setup}

The experimental setup in Figure 6 was designed by Stander and Heyns [24] and was refurbished to perform gear fatigue tests under fluctuating conditions. The electrical motor supplies rotational energy to the system and the alternator, which is connected to a resistor bank, dissipates the energy from the system by applying a counteracting load to the motor. A personal computer is used to control the rotational speed and the load in the system. Three gearboxes are present in this setup, with the gearbox that is monitored with accelerometers labelled as the monitored gearbox. The input shaft of the monitored gearbox contains a zebra tape shaft encoder, with 88 PPR, and an optical probe. The output of the monitored gearbox contains a proximity probe that is triggered by the shaft key and produces 1 PPR.

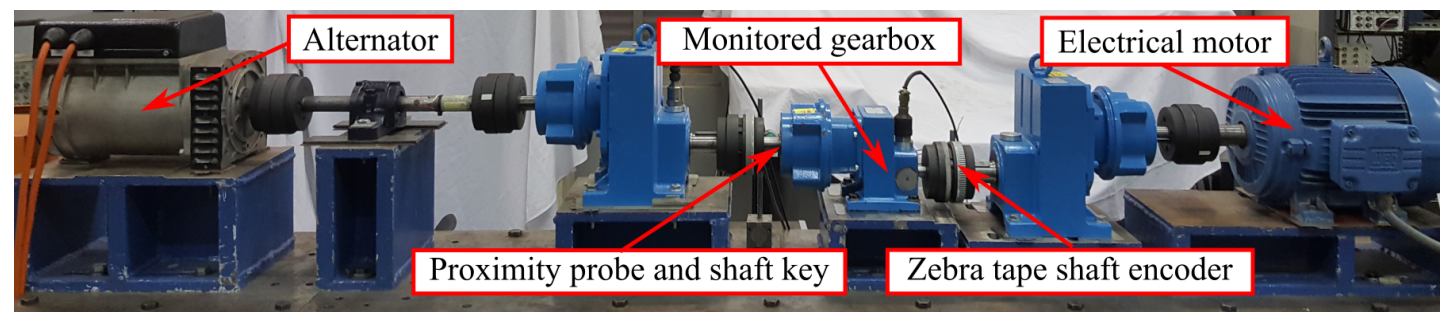

Figure 6: Experimental setup

The axial vibration signal, obtained from the tri-axial accelerometer that is on the bearing housing of the monitored gearbox, is used to extract the rotational information from. The axial vibration signal is used because the helical gears cause strong axial excitations which result in prominent meshing components in its vibration spectrum. The tachometer signals are both sampled at $51.2 \mathrm{kHz}$ and the acceleration signal is sampled at a rate of $25.6 \mathrm{kHz}$ with an OROS OR35 data acquisition device.

Three rotational speed profiles, shown in Figure 7, were applied to the system during which vibration measurements and tachometer measurements were made. The unique rotational speed profiles contained relatively large rotational speed fluctuations to validate the proposed methodology. The tachometer signal that is associated with the 88 PPR zebra tape shaft encoder, geometrically compensated with the technique proposed by Diamond et al. [6], is used to compute the rotational speeds in Figure 7 as well as the analytical phase of the experimental data. 
(a)

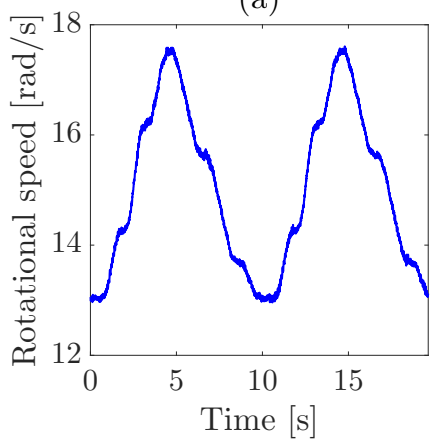

(b)

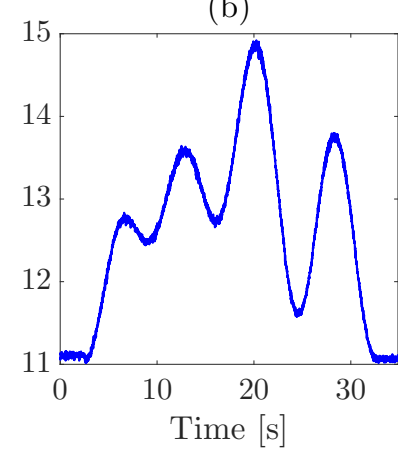

(c)

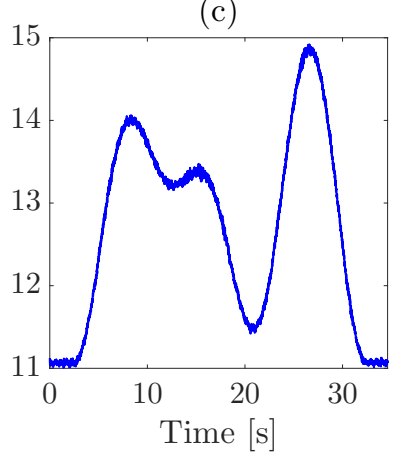

Figure 7: Experimental rotational speed profiles with Profile 1 indicated by (a), Profile 2 indicated by (b) and Profile 3 indicated by (c) in this figure.

\subsubsection{Maxima tracking and phase estimation results}

The performance of the maxima tracking algorithms is evaluated on the experimental data in this section, where a similar process to the numerical investigation is followed. The $\Delta f_{c}$ is chosen to be equal to 6.4 times the frequency resolution of the spectrogram and the relative bandwidth of the VKF is $10^{-4}$ on all investigated cases. The spectrogram of the vibration signal associated with the first rotational speed profile (see (a) in Figure 7) is superimposed with the results of the two maxima tracking approaches in Figure 8i, where the benchmark and the proposed method are indicated by (a) and (b), respectively. The gear mesh frequencies of the monitored gearbox

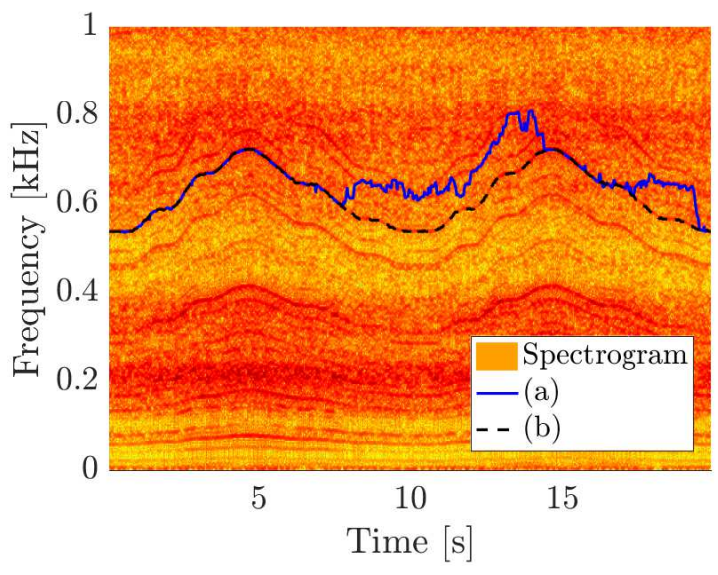

(i)

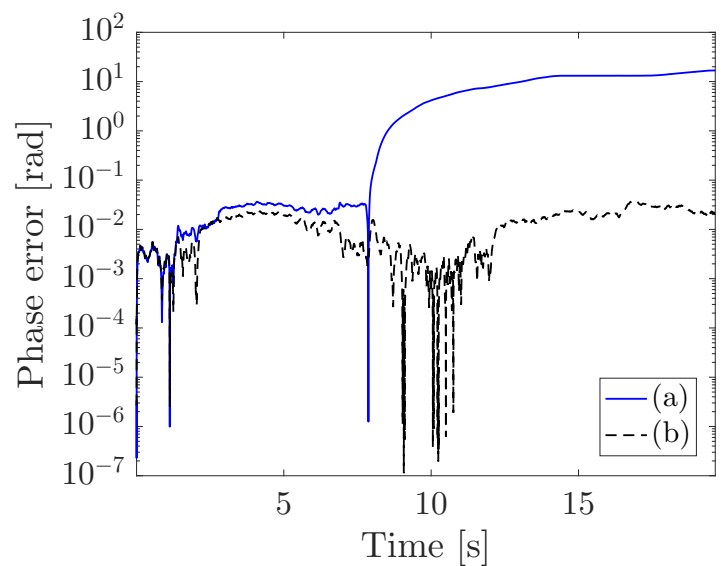

(ii)

Figure 8: The spectrogram of the vibration signal from the first experimental rotational speed profile is superimposed with the maxima tracking results in Figure 8i. The resulting phase estimation errors of the two results are compared in Figure 8ii. Note that the benchmark maxima tracking method's results are indicated by (a) and the results from the proposed method are indicated by (b).

are tracked in Figure 8i, however it is immersed in noise induced by the resonance bands and 
other frequency components. This adversely affects the performance of the benchmark maxima tracking algorithm in the regions with large frequency gradients as seen in Figure 8i. In contrast, the proposed maxima tracking algorithm, is able to track the correct frequency component and resulted in a significantly better phase estimate as shown in Figure 8ii. The large phase errors associated with the benchmark method is attributed to the maxima tracking algorithm drifting off from the true estimate.

The spectrogram of the vibration signal, associated with the second rotational speed profile (see (b) in Figure 7), is shown in Figure 9i. The results from the maxima tracking processes are superimposed in Figure 9i, where it is seen that both algorithms are able to track the component of interest. The resulting phase errors of the two maxima tracking approaches used

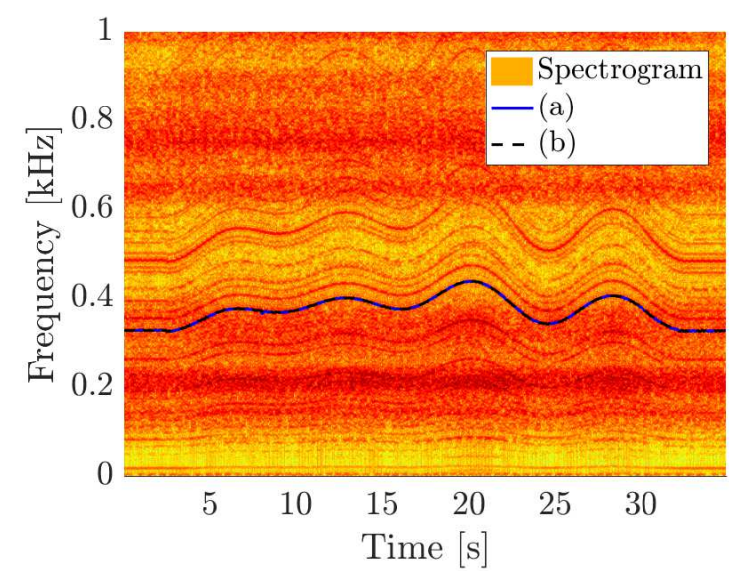

(i)

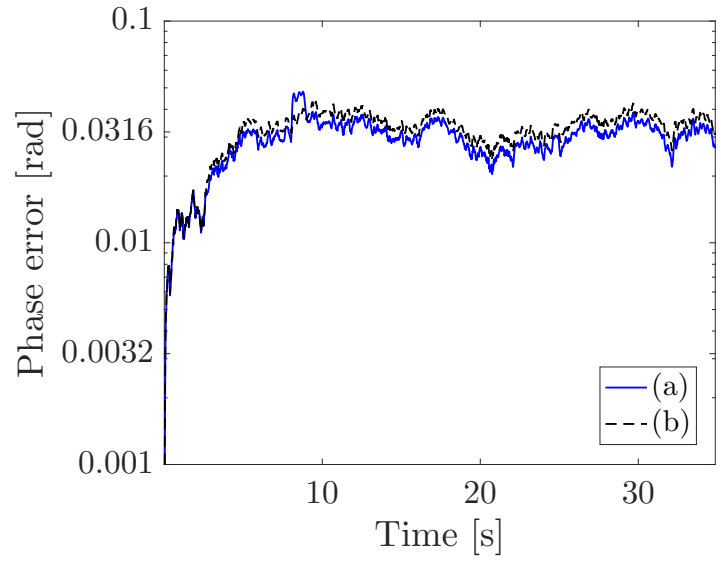

(ii)

Figure 9: The spectrogram and the maxima tracked results are presented in Figure 9i with the resulting phase errors for the benchmark and the proposed maxima tracking algorithms in Figure 9ii using the proposed tacholess order tracking method. This is performed for the second experimental signal shown as (b) in Figure 7.

in the proposed tacholess order tracking method, presented in Figure 9ii, confirms both maxima tracking algorithms performed well. Even though the benchmark maxima tracking algorithm resulted in the maximum phase error, on average it performs slightly better.

The spectrogram of the last experimental vibration signal, associated with profile (c) in Figure 7, is superimposed with the maxima tracking results of the two algorithms illustrated in Figure 10i. The resulting phase errors are shown in Figure 10ii, where it can be seen that the proposed maxima tracking method performs significantly better than the benchmark maxima tracking algorithm.

The results of the three experimental profiles are summarised in Table 3. The signal-to- 


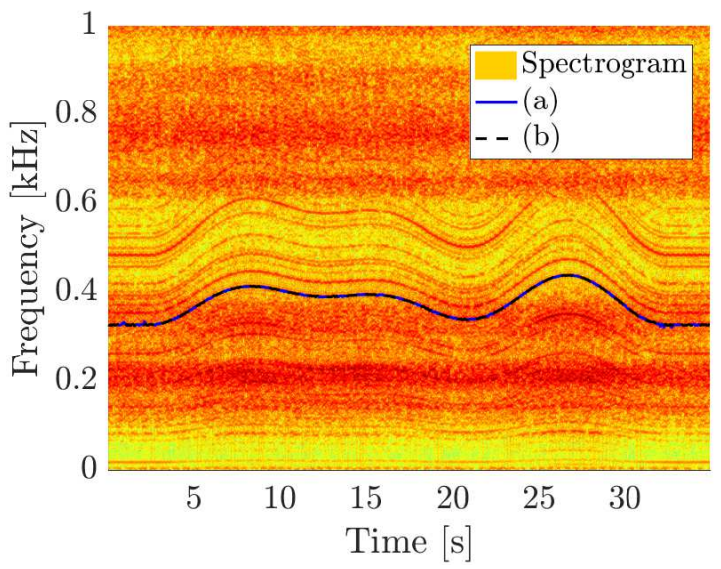

(i)

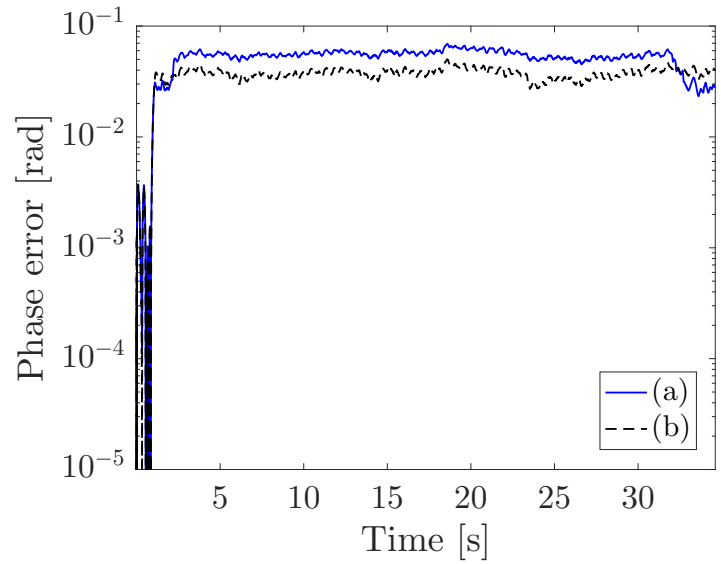

(ii)

Figure 10: The spectrogram and the maxima tracked results are presented in Figure 10i with the resulting phase errors for the benchmark and the proposed maxima tracking algorithms in Figure 10ii using the proposed tacholess order tracking method. This is performed for the third experimental signal shown as (c) in Figure 7.

noise ratio is calculated with Equation (39) and is presented in table 3. The relative phase

Table 3: Summary of the maxima tracking results from the experimental rotational speed profiles. All the phase units are in radians, except the maximum relative phase error which is in $\%$.

\begin{tabular}{lccc}
\hline & Profile 1 & Profile 2 & Profile 3 \\
\hline Maximum phase completed & 295.2322 & 439.753 & 439.7533 \\
Signal-to-noise ratio (db) & 19.35 & 20.49 & 18.04 \\
Benchmark method by [10]: & & & \\
$\quad$ RMS phase error & 8.3951 & 0.030019 & 0.053025 \\
$\quad$ Maximum phase error & 16.8094 & 0.048112 & 0.069044 \\
$\quad$ Maximum relative phase error (\%) & 5.6936 & 0.010941 & 0.015701 \\
Proposed method & & & \\
$\quad$ RMS phase error & 0.016662 & 0.033053 & 0.037182 \\
$\quad$ Maximum phase error & 0.037697 & 0.04343 & 0.050224 \\
$\quad$ Maximum relative phase error (\%) & 0.012768 & 0.009876 & 0.011421 \\
\hline
\end{tabular}

error of the proposed tacholess order tracking method, calculated from Equation (33) for the different experimental signals, is very small. The proposed maxima tracking algorithm results in significant improvements in profile 1 and profile 3, and performs almost equally well with the benchmark maxima tracking algorithm on profile 2 .

\subsubsection{Tacholess order tracking parameters}

The parameters used in the tacholess order tracking process for the numerical and the experimental datasets are presented in Table 4 with the caption describing the parameters. 
Table 4: The table contains the parameters that were used in the numerical (Num.) and experimental (Exp.) analyses. The sampling frequency of the vibration signal $f_{s}$; the window length, window overlap, zero padding and window type that are used for the spectrogram; The number of data points $N_{m}$ and the order of the polynomial $N_{p}$ used in the maxima tracking process; $c_{s}$ and $k$ which are used to calculate the instantaneous phase; $\Delta f_{c}$ describes the feasible region of the maxima tracking process; $\rho$ is used in the maxima tracking optimisation process; $B_{w}$ is used in the Vold-Kalman filter.

\begin{tabular}{cccccccc}
\hline & Unit & Num. 1 & Num. 2 & Num. 3 & Exp. 1 & Exp. 2 & Exp. 3 \\
\hline$f_{s}$ & Hz & 10000 & 10000 & 1500 & 25000 & 25000 & 25000 \\
Window length & Samples & 4000 & 4000 & 750 & 3500 & 5000 & 5000 \\
Window overlap & Samples & 3600 & 3600 & 675 & 2800 & 4000 & 4000 \\
Zero padding & Samples & 4192 & 4192 & 1298 & 4692 & 11384 & 11384 \\
Window type & - & Rect. & Rect. & Rect. & Rect. & Rect. & Rect. \\
$N_{m}$ & - & 5 & 5 & 5 & 5 & 5 & 5 \\
$N_{p}$ & - & 1 & 1 & 1 & 1 & 1 & 1 \\
$c_{s}$ & - & 1 & 1 & 40 & 37 & 37 & 37 \\
$k$ & - & 1 & 1 & 3 & 7 & 5 & 5 \\
$\Delta f_{c}$ & $\mathrm{~Hz}$ & 20 & 20 & 2 & 20 & 10 & 10 \\
$\rho$ & - & $10^{9}$ & $10^{9}$ & $10^{9}$ & $10^{9}$ & $10^{9}$ & $10^{9}$ \\
$B_{w}$ & - & 0.1 & 0.1 & 0.0001 & 0.0001 & 0.0001 & 0.0001 \\
\hline
\end{tabular}

\subsubsection{Order tracking results}

The instantaneous phase is used in the previous sections to quantify the performance of the proposed tacholess order tracking method with respect to using the tachometer signal obtained from the zebra tape shaft encoder and the optical probe. The instantaneous phase is used to resample the vibration signal with a constant angular frequency as illustrated by (f) in Figure 1. In this section the spectrum of the order tracked signals, using the proposed tacholess order tracking method and the tachometer signal from the zebra tape shaft encoder are compared. The linear amplitude spectrum of the two approaches are compared in Figure 11 for the first experimental profile (See (a) in Figure 7).

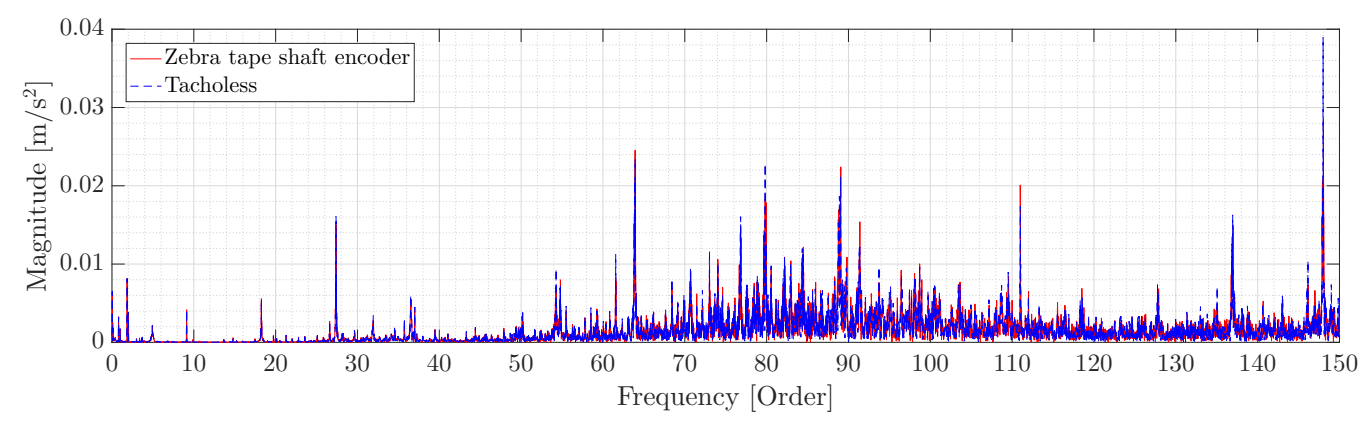

Figure 11: The linear spectrum of the order tracked vibration signal using the two order tracking approaches.

The resulting spectrum using the tacholess and the tacho-based approaches, in Figure 11, 
are very similar. A more detailed view of the lower frequency spectrum is shown in Figure 12 for the same order tracked signal as used in Figure 11. The frequency components of the shafts

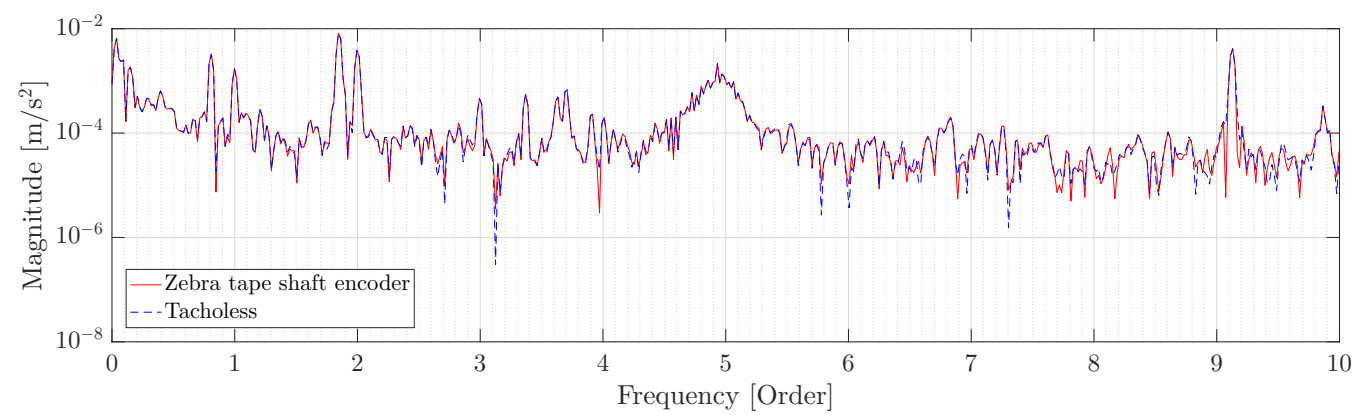

Figure 12: The 0 -10 order spectrum of the order tracked vibration signal using the two order tracking approaches.

and the gear mesh frequencies are easily identified in the spectrum with clear and distinct peaks. The results in Figure 11 and in Figure 12, attest to the ability of the proposed method to order track the measured vibration signal without using a tachometer.

\subsubsection{Rotational speed estimation}

The last aspect that is briefly investigated in this study is the ability of the proposed method to estimate the rotational speed of the shaft of interest. The normalised maxima tracked frequency, $f_{\max } /\left(c_{s} \cdot k\right)$, and the derivative of the Hilbert transform phase is superimposed with the rotational speed calculated from the tachometer signal associated with the zebra tape shaft encoder in Figure 13. It is evident from the results that the derivative of the instantaneous phase
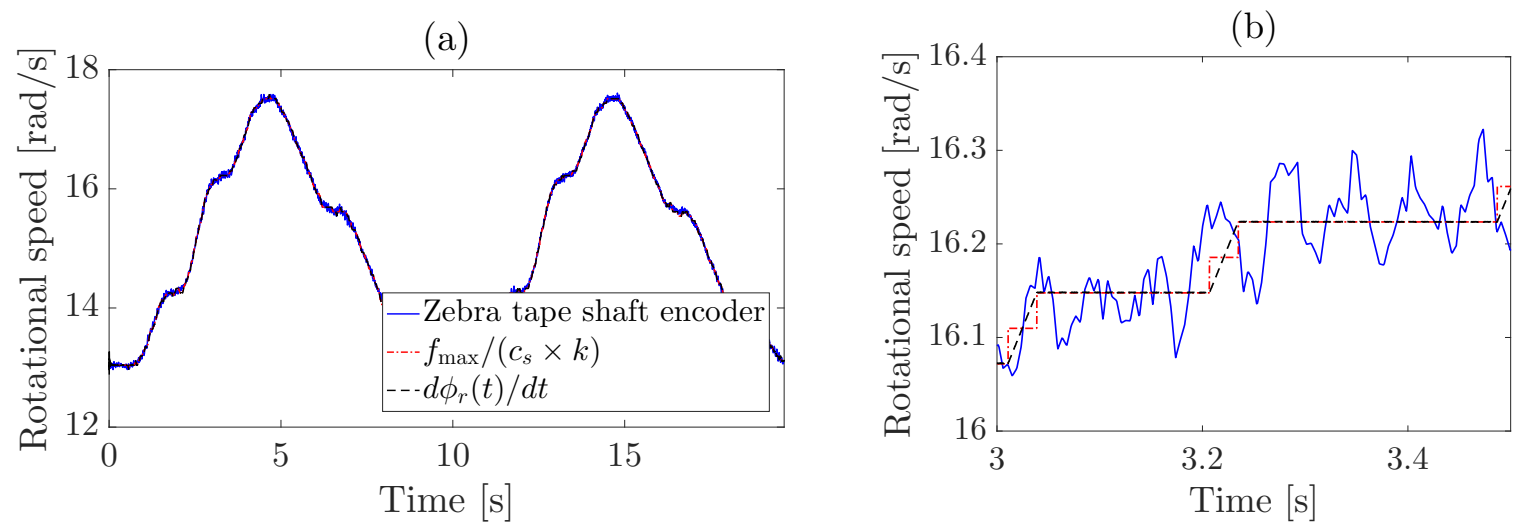

Figure 13: The rotational speed estimates of three approaches on the first experimental speed profile. The full view is shown in (a), while a zoomed view is shown in (b).

of the shaft, obtained from the Hilbert transform, and the maxima tracked frequency performs well in estimating the rotational speed of the shaft. The RMS error, obtained from the esti- 
mated rotational speed by $f_{\max } /\left(c_{s} \cdot k\right)$ is $0.0423 \mathrm{rad} / \mathrm{s}$. The RMS error of the rotational speed obtained from the Hilbert transform is $0.0425 \mathrm{rad} / \mathrm{s}$, which is slightly higher than the $f_{\max } /\left(c_{s} \cdot k\right)$ estimate due to the end point effects of the Hilbert transform. The estimated rotational speed does not contain diagnostic information similar to the zebra tape shaft encoder signal, however it provides invaluable information as well. This information can assist with prognostics and the maintenance task in general, since the number of cycles completed by the different components in certain operating regimes can be estimated.

\section{Conclusions and recommendations}

The proposed tacholess order tracking method is validated on three numerical and three experimental datasets. The instantaneous phase, which is used to resample the vibration signal from the time to the angle domain, is accurately estimated with the proposed method on the six datasets. The results also indicate that it is more robust to noise and other frequency components than the benchmark maxima tracking algorithm. The low phase estimation errors result in very good order tracking results, which is the main objective when applying this technique. It is also possible to accurately estimate the mean rotational speed with the proposed tacholess order tracking method, which can for example be used to support maintenance decisions and can be used in the remaining useful life estimation process of the rotating machine components.

It is however suggested that the technique needs to be employed on datasets, obtained from industrial environments so that it can fully be validated. It is recommended that techniques need to be explored which can help to estimate a good initial value of the IF and the $k \cdot c_{s}$ term so that the tacholess order tracking process can automatically be performed. The characteristics of the angular-displacement Vold-Kalman filter can be investigated further as well to ensure that the performance is optimal for a broad range of applications. Incorporating acceleration information into the process can also be investigated with multi-order techniques in future investigations, because it can lead to even more robust tacholess order tracking techniques.

\section{Acknowledgements}

The authors gratefully acknowledge the support that was received from the Eskom Power Plant Engineering Institute (EPPEI) in the execution of the research. 


\section{References}

[1] J. Antoni, F. Bonnardot, A. Raad, M. El Badaoui, Cyclostationary modelling of rotating machine vibration signals, Mechanical Systems and Signal Processing 18 (6) (2004) 12851314.

[2] T. Heyns, P. S. Heyns, J. P. De Villiers, Combining synchronous averaging with a Gaussian mixture model novelty detection scheme for vibration-based condition monitoring of a gearbox, Mechanical Systems and Signal Processing 32 (2012) 200-215.

[3] C. J. Stander, P. S. Heyns, W. Schoombie, Using Vibration Monitoring for Local Fault Detection on Gears Operating Under Fluctuating Load Conditions, Mechanical Systems and Signal Processing 16 (6) (2002) 1005-1024.

[4] X. Qi, Z. Yuan, X. Han, Diagnosis of misalignment faults by tacholess order tracking analysis and RBF networks, Neurocomputing 169 (2015) 439-448.

[5] D. Abboud, J. Antoni, S. Sieg-Zieba, M. Eltabach, Envelope analysis of rotating machine vibrations in variable speed conditions: A comprehensive treatment, Mechanical Systems and Signal Processing 84 (2017) 200-226.

[6] D. H. Diamond, P. S. Heyns, A. J. Oberholster, Online shaft encoder geometry compensation for arbitrary shaft speed profiles using Bayesian regression, Mechanical Systems and Signal Processing 81 (2016) 402-418.

[7] K. Fyfe, E. Munck, Analysis of computed order tracking, Mechanical Systems and Signal Processing 11 (2) (1997) 187-205.

[8] F. Bonnardot, M. El Badaoui, R. B. Randall, J. Danière, F. Guillet, Use of the acceleration signal of a gearbox in order to perform angular resampling (with limited speed fluctuation), Mechanical Systems and Signal Processing 19 (4) (2005) 766-785.

[9] Q. Leclère, H. André, J. Antoni, A multi-order probabilistic approach for Instantaneous Angular Speed tracking debriefing of the CMMNO'14 diagnosis contest, Mechanical Systems and Signal Processing 81 (2016) 375-386. 
[10] J. Urbanek, T. Barszcz, J. Antoni, A two-step procedure for estimation of instantaneous rotational speed with large fluctuations, Mechanical Systems and Signal Processing 38 (1) (2013) 96-102.

[11] F. Combet, L. Gelman, An automated methodology for performing time synchronous averaging of a gearbox signal without speed sensor, Mechanical Systems and Signal Processing 21 (6) (2007) 2590-2606.

[12] M. Zhao, J. Lin, X. Wang, Y. Lei, J. Cao, A tacho-less order tracking technique for large speed variations, Mechanical Systems and Signal Processing 40 (1) (2013) 76-90.

[13] J. Lin, M. Zhao, A review and strategy for the diagnosis of speed-varying machinery, in: Prognostics and Health Management (PHM), 2014 IEEE Conference on, IEEE, 2014, pp. $1-9$.

[14] C. J. Stander, P. S. Heyns, Transmission path phase compensation for gear monitoring under fluctuating load conditions, Mechanical Systems and Signal Processing 20 (7) (2006) $1511-1522$.

[15] K. C. Gryllias, I. A. Antoniadis, Estimation of the instantaneous rotation speed using complex shifted Morlet wavelets, Mechanical Systems and Signal Processing 38 (1) (2013) 78-95.

[16] T. Heyns, P. S. Heyns, R. Zimroz, Combining discrepancy analysis with sensorless signal resampling for condition monitoring of rotating machines under fluctuating operations, Ninth International Conference on Condition Monitoring and Machinery Failure Prevention Technologies 2 (2) (2012) 52-58.

[17] G. He, K. Ding, W. Li, X. Jiao, A novel order tracking method for wind turbine planetary gearbox vibration analysis based on discrete spectrum correction technique, Renewable Energy 87 (2016) 364-375.

[18] Z. K. Peng, F. L. Chu, Application of the wavelet transform in machine condition monitoring and fault diagnostics: A review with bibliography, Mechanical Systems and Signal Processing 18 (2) (2004) 199-221.

[19] C. M. Bishop, Pattern recognition and machine learning, Springer, 2009. 
[20] J. S. Arora, Introduction to optimum design, Elsevier/Academic Press, 2011.

[21] J. Tuma, Setting the passband width in the vold- kalman order tracking filter, in: Twelfth International Congresson Sound and Vibration, Lisbon, 2005, pp. 1-8.

[22] M.-C. Pan, Y.-F. Lin, Further exploration of Vold-Kalman-filtering order tracking with shaft-speed information - I: Theoretical part, numerical implementation and parameter investigations, Mechanical Systems and Signal Processing 20 (5) (2006) 1134-1154.

[23] J. R. Blough, A survey of DSP methods for rotating machinery analysis, what is needed, what is available, Journal of Sound and Vibration 262 (2003) 707-720.

[24] C. J. Stander, P. S. Heyns, Instantaneous angular speed monitoring of gearboxes under noncyclic stationary load conditions, Mechanical Systems and Signal Processing 19 (4) (2005) $817-835$. 